\title{
Levantamento do Ensino de Engenharia de Requisitos e Cursos à Distância no Brasil
}

\author{
Survey of requirements engineering teaching in Brazilian E-Learning of the courses
}

Luiz Felipe Dias da Costa ${ }^{1}$ (i) orcid.org/0000-0001-6635-4686

João Henrique Correia Pimentel ${ }^{2}$ (i) orcid.org/0000-0002-7441-0796

Maria Lencastre Pinheiro de Menezes Cruz ${ }^{2}$ (iD) orcid.org/0000-0002-8032-8801

${ }^{1}$ Escola Politécnica de Pernambuco, Universidade de Pernambuco, Recife, Brasil

E-mail do autor principal: Luiz Felipe Dias da Costa felipe.dias.ads@gmail.com

\section{Resumo}

A educação à distância (EAD) cada vez vem tendo maior aceitação na sociedade e, consequentemente, sendo intensivamente implantada nas instituições de ensino em vários cursos. O crescimento dessa modalidade de ensino está associado ao avanço nas tecnologias de comunicação para auxiliar as metodologias de ensino não-presenciais, somado ao amadurecimento decorrente de experiências bem-sucedidas. Este trabalho tem a finalidade de fazer um levantamento do ensino de Engenharia de Requisitos (ER) nos cursos de EAD na área de Tecnologia da Informação (TI) existentes no Brasil, em instituições federais e estaduais. Esta pesquisa é o ponto de partida para novos estudos, como: a avaliação da forma de ensino de ER, padronização de conteúdo e análise de qualidade nas disciplinas de Engenharia de Requisitos à distância.

Palavras-Chave: Educação à Distância; Engenharia de Requisitos; Engenharia de Software; Ensino de Engenharia de Software.

\begin{abstract}
Distance education (E-learning) is becoming more widely accepted in society and, consequently, it has been intensively adopted in educational institutions. However, it is important to take into account the physical distance of teacher/student, which imposes limitations on the educational process. Thus, it is important to rethink how this teaching should be done mainly for disciplines involving practice. This article aims to do a survey on E-learning courses in the Information Technology (IT) field in Brazil, at federal and state institutions, focusing on Requirements Engineering teaching. This research is the starting point for future studies such as: evaluation of the teaching methods, content standardization, and quality analysis in the disciplines of distance learning for Requirements Engineering.
\end{abstract}

Key-words: E-learning; Requirements Engineering; Software Engineering; Software Engineering Teaching. 


\section{Introdução}

Educação à distância (EAD) é o processo de ensino-aprendizagem, onde professores e alunos geralmente não estão juntos fisicamente, mas estão conectados, mediados por tecnologias como a Internet. O EAD é uma modalidade de ensino que tem se tornado cada vez mais comum, expandindo possibilidades na formação profissional e científica. A educação à distância atende assim a uma necessidade social, a partir do uso de tecnologias da informação e comunicação, que proporcionam interatividade entre o aluno e o professor. O aluno deve gerenciar o seu tempo de estudo, e a autoaprendizagem é estimulada, com a mediação de recursos didáticos disponíveis e sistematicamente organizados [1].

Cada vez mais aumenta a aceitação da modalidade de ensino à distância, que propõe atender as pessoas que buscam e precisam de praticidade, que moram longe das instituições de ensino, ou que trabalham.

Nos cursos de TI (Tecnologia da Informação), que são fundamentados no desenvolvimento de sistemas, geralmente é abordado o assunto de Engenharia de Requisitos (ER). Este conteúdo é responsável pelo levantamento de informações para o projeto/implementação de um sistema de qualidade, com controle e manutenção [2]. O conteúdo de ER já está presente na forma EAD em várias instituições, porém, não se tem disponível o quantitativo de instituições federais e estaduais que têm essa disciplina ofertada no formato EAD; consequentemente, não se sabe como ela vem sendo aplicada, seus problemas e pontos de sucesso.

Este artigo tem como objetivo realizar um levantamento quantitativo das instituições que têm ensino à distância e trabalham com cursos de TI, assim como onde e como abordam a ER. Através dessa pesquisa exploratória, espera-se coletar e organizar essas informações e levá-las a público, contribuindo com possíveis pesquisas futuras sobre o ensino a distância para melhorar/analisar a qualidade da disciplina de ER.

Este artigo está estruturado da seguinte forma. A seção 2 apresenta a metodologia aplicada para 0 levantamento nas instituições de ensino. A seção 3 descreve alguns conceitos relacionados ao Ensino à Distância. Em seguida, a seção 4 apresenta conceitos da Engenharia de Requisitos e a sua forma de ensino. Já a seção 5 e 6 apresenta o resultado do levantamento realizado. A seção 7 faz algumas conclusões finais e indica trabalhos futuros. Por fim, um apêndice mostra uma tabela da pesquisa realizada.

\section{Metodologia}

Para atender ao objetivo central desta pesquisa, foram utilizadas as estratégias e passos descritos a seguir.

No embasamento teórico dos termos, conceitos e definições da Engenharia de Requisitos e seu ensino, foi realizado um levantamento em livros e artigos [3, $4,5,6]$.

$\mathrm{Na}$ pesquisa relativa às instituições de ensino, foi usada a Internet como fonte de dados de pesquisa em sites. Foram enviadas mensagens via e-mail para coordenadores de cursos visando levantar dados mais específicos. À medida que os dados foram sendo coletados, em paralelo, foi organizada e alimentada uma tabela com os dados colhidos.

Para complementar o levantamento, foi elaborado um questionário, para ser enviado para algumas instituições de ensino, a fim de investigar: como a ER vem sendo aplicado nos cursos de TI, a forma como é trabalhada, e o nível de satisfação dos coordenadores em relação à aplicação ao conteúdo de ER que são lecionadas na modalidade de ensino à distância. Dos questionários enviados, apenas duas intuições preencheram e responderam o questionário.

Foi feita uma análise dos resultados coletados, e então estes foram organizados e estruturados, facilitando o entendimento para a obtenção das informações desejadas com as possíveis diretrizes.

Pode se observar que na metodologia definida, para entender os resultados obtidos e na tentativa de interpretar as possíveis diretrizes que provocaram o resultado encontrado, foi utilizada uma abordagem qualitativa. Foram quantificados os dados pesquisados, traduzidos os números em opiniões e informações para serem classificados e analisados, apresentando em formato de porcentagem.

\section{A Educação à Distância}

A educação trata de aspectos de relações pessoais e sociais. É um processo de desenvolvimento intelectual humano de ensinar, aprender, pensar, criar, construir conhecimentos [7]. A educação é um processo contínuo ou continuado de formação 
constante de aprender, com teoria ou prática, por situações presenciadas ou vividas de cada indivíduo ao longo da vida para evolução das faculdades física, moral e intelectual. O processo educativo contínuo é a busca pela melhoria de qualidade na formação docente e discente; porém, não há uma fórmula pronta a seguir, a fórmula é criada, desvendada a cada passo, de acordo com experiências vivenciadas nas várias formas de educar. [8]

A EAD é um formato organizado de aprendizagem e autodisciplinar, baseadas na separação física dos aprendizes e os envolvidos na organização de sua aprendizagem. A separação pode aplicar-se em todo ou parte do processo de aprendizagem [9].

O avanço da educação à distância está associado ao crescimento de técnicas de comunicação para auxiliar no processo de ensino não presenciais; esse impulsiona novas experiências, e consequentemente, contribui para o crescimento e amadurecimento da educação à distância. [9, 10] Essas técnicas são métodos de transmitir conhecimentos e habilidades, mediante a divisão de trabalho entre os profissionais responsáveis pelas técnicas a serem usadas; o objetivo é reproduzir material de ensino de qualidade, o que torna possível instruir um grande número de alunos ao mesmo tempo, independentemente de onde eles vivem. É uma aprendizagem planejada que geralmente ocorre num lugar diferente do ensino e, por causa disso, requer técnicas para comunicação mediadas por recursos didáticos e sistematicamente organizados. [7]

A modalidade EAD no Brasil ganhou força com a popularização da banda larga no país. O crescimento de novos polos de ensino e a criação de novos cursos, somado com uma nova geração de jovens nascidos em um ambiente cem por cento digital, abre novas perspectivas, facilitando o acesso dos alunos ao ensino superior. [11]

A definição de EAD é influenciada pela distância, devendo ser compreendida basicamente como a separação espacial (geográfica/local) entre participantes do processo educacional, sejam estes alunos ou professores. Em aulas por videoconferência é comum que os alunos estejam juntos, mas em lugar diferente do professor. Por outro lado, quando o estudo ocorre pela internet, é comum alunos e professores estejam em locais diferentes e acessem o curso e os materiais e recursos didáticos em momentos diferentes. Estes dois exemplos ilustram a existência de diferentes possibilidades de distanciamento entre alunos e professores. [12]

Algumas características são atrativas aos adeptos da modalidade EAD, como por exemplo: terem acesso a informações a qualquer momento e em qualquer lugar; nos momentos em que têm motivação para estudar; o uso de aplicativos e softwares e recursos auxiliam na comunicação e na troca de informações (como chats, fóruns, e-mail, plataformas de transferência de arquivos e gerenciamento de conteúdos). [13]

A disponibilização de informações e iteração com tutores, através de ambientes virtuais, permite que os alunos acessem os conteúdos sempre que quiserem, publiquem suas dúvidas e troquem conhecimentos a qualquer hora, em qualquer lugar. [13]

O custo para realizar um curso à distância é inferior aos presenciais, isso ocorre devido à ausência da necessidade de uma estrutura física, essencial nos cursos presenciais (mesas, cadeiras, quadro, projetores, entre outros). Além disso, não é necessário o deslocamento para a instituição de ensino, o aluno pode realizar atividades em casa ou em outro lugar que seja cômodo para ele. [14]

De acordo com o MEC (2016) "Resolução CNE/CES no 1 ", os cursos superiores, na modalidade EAD, devem cumprir, rigorosamente, as Diretrizes e Normas e as Diretrizes Curriculares Nacionais dos cursos de graduação. Para serem ofertados cursos superiores, as instituições de ensino devem ser aderentes à política institucional das IES (Instituições de Ensino Superior) do Ministério da Educação (MEC), que é o órgão federal responsável por credenciar, autorizar e avaliar todas as instituições de ensino superior do país, tanto no ensino presencial quanto à distância[15]. A garantia que a graduação pelo ensino presencial e à distância são a mesma, é alcançada por ambas as modalidades estarem submetidas à Lei de Diretrizes e Bases da Educação, e têm que ser submetidas à avaliação de igual modo[15]. Portanto, não importa a forma que o aluno aprende, e sim que aprende o mesmo conteúdo; com isso, os diplomas de um curso superior tradicional têm a mesma validade dos cursos EAD.

O perfil do aluno tem que ser adequado à metodologia de ensino à distância; ele precisa estar comprometido com o estudo, pois este requer muita dedicação, disciplina e responsabilidade, uma vez que não existe a presença física do professor a cobrar. A concentração do aluno pode ser um grande desafio; diferentes formas de lazer, conteúdo, entretenimentos estão disponíveis e são facilmente acessíveis, sendo obstáculos à concentração natural; assim, é preciso ter foco. O controle do tempo pode ser considerado uma vantagem, mas também pode ser um desafio uma vez que o próprio aluno tem que fazer e seguir

http: / / dx.doi.org/10.25286/repa.v2i2.561 
os seus horários de estudos. O aluno que não consegue administrar seu tempo, com as atividades corriqueiras e os estudos de forma correta, acaba comprometendo seu rendimento na aprendizagem. É importante também não deixar acumular tarefas e exercícios e acompanhar as aulas com regularidade; para isso, é relevante que seja montado um roteiro a ser seguido e um cronograma para que as os estudos sejam executados.

\section{Engenharia de Requisitos}

Muitos observadores da indústria de software, já na década de 70, caracterizavam os problemas associados ao desenvolvimento de software como uma "crise". [16] As investigações determinaram que esses problemas, normalmente, não têm uma causa única, mas as deficiências nos requisitos dos sistemas contribuem fortemente para o problema. Para muitos desenvolvedores de sistemas de software complexos e grandes, os requisitos são o maior problema da Engenharia de Software. Nenhuma outra atividade do processo de desenvolvimento é tão difícil de executar ou tão desastrosa em seus resultados, quando feita de forma inadequada. [17]

O desenvolvimento de um software é de natureza complexa pois além de trabalhar com pessoas, cada cenário exige soluções variadas em cada contexto. As etapas da Engenharia de Requisitos são a base de um projeto e, dependendo de como forem executadas, há grande chance de sucesso ou não na solução do projeto. [18]

O processo de ER é um conjunto de atividades bem definidas e distribuídas aos responsáveis pela sua execução; documentos e ferramentas são muito utilizados nesse processo. É definido como a equipe deverá trabalhar para atingir os objetivos, o processo de comunicação e interação que será utilizado, para se poder cumprir os prazos e desenvolver um software de qualidade de acordo com os requisitos definidos. [18]

A ER pode ser definida como um processo relevante do desenvolvimento de um sistema, que contempla o levantamento de informações de forma iterativa e cooperativa sobre a análise, observações de documentação resultantes a realidade de um problema. [3] Assim, a ER, uma etapa da Engenharia de Software, foi criado para cobrir todas as atividades envolvidas no descobrimento, documentação e manutenção de um conjunto de requisitos para um sistema baseado em computador. O uso do termo engenharia implica em técnicas sistemáticas e repetitivas a serem usadas para assegurar que os requisitos especificados de um sistema sejam completos, consistentes, relevantes e claros. [3]
Sob o ponto de vista conceitual, no contexto das disciplinas de Engenharia de Requisitos, são abordados os seis principais fundamentos, diferentes tipos de processos e técnicas aplicáveis. [3]

As atividades da ER são fundamentais no processo de desenvolvimento de software. Por se tratarem de atividades de grande importância no ciclo de vida do software, e que se relacionam diretamente com a qualidade do produto a ser desenvolvido, a ER precisa ser devidamente planejada. As etapas de ER ajudam no entendimento de como será o processo definir quais as necessidades do cliente e como os usuários finais vão interagir com o software. A ER é composta por sete etapas distintas: concepção, levantamento, elaboração, negociação, especificação, validação e gestão. Todas as etapas são adequadas de acordo com a necessidade do cliente. [4]

Concepção: a iniciação de um projeto de software ocorre quando se identifica a necessidade de um software para solução de um problema em um contexto específico. Ela é a primeira etapa, onde se procura definir o escopo e a natureza do problema. Em uma conversa informal com os interessados no software é definido o plano de negócio e avaliada a possibilidade do suposto software ser desenvolvido. As informações iniciais podem sofrer mudanças, porém elas são importantes para produzir discussões com a equipe de engenharia de software. $\mathrm{Na}$ concepção do projeto é estabelecido um entendimento básico do problema, onde é esperado que a solução desejada seja feita em colaboração com os demais interessados e a equipe que será encarregada pelo software. [4]

Levantamento: fase onde se pergunta ao cliente, usuários e os demais envolvidos (stakeholders), quais são as funcionalidades que o sistema deve ter, as regras de negócio dessas funcionalidades, restrições, usabilidade, para que cada um seja atendido de acordo com a necessidade da empresa. Não é uma tarefa fácil. É preciso mobilizar os envolvidos para que estes compartilhem informações e ajudem a estabelecer metas e prioridades. [4] Alguns problemas podem ser encontrados durante o processo de levantamento de requisitos, entre eles: limites de escopo não definidos de forma clara, clientes/usuários descrevem detalhes técnicos que confundem ao invés de esclarecer. As especificações de requisitos mudam com o passar do tempo, os clientes/usuários não estão certos do que realmente precisam, ou não entendem a necessidade e importância da especificação dos requisitos, ocorrendo um grande risco de se resolver o problema errado. Para evitar esses problemas é preciso realizar a atividade de levantamento de requisitos de forma organizada. [4, 5] 
Elaboração: esta etapa é iniciada após o cliente aprovar a proposta especificada. As informações colhidas com os clientes, durante a concepção e o levantamento, são expandidas e aprimoradas durante a elaboração. [4, 5] A elaboração é realizada através de criação e refinamento de cenários de usuários que descrevem como o usuário final irá interagir com o sistema. Esses cenários são analisados sintaticamente para extrair classes, atributos das classes, e os serviços exigidos por essas classes são identificados. Também se identificam as relações e colaboração entre as classes, além de uma variedade de diagramas que são produzidos. Nesse processo é recomendada a realização de oficinas/workshop com participação ativa e massiva das partes interessadas. Alguns mecanismos são usados para melhor entendimento do cliente, como a elaboração de protótipos. $[4,5]$ Com os requisitos destrinchados, os requisitos-funcionais podem ser descritos através de documentos de caso de uso. [5]

Negociação: esta etapa é responsável por conciliar e resolver conflitos que possam existir. Os conflitos têm que ser resolvidos por meio da negociação. Deve ser solicitado aos envolvidos que eles ordenem as prioridades das funcionalidades e discutam sua prioridade; para isso devem avaliar aspectos como o custo e risco até que se chegue a um acordo (os requisitos sejam retirados, combinados e/ou modificados, de modo que cada parte atinja um grau de satisfação). [4]

Especificação: esta etapa deve demonstrar os requisitos solicitados pelo cliente. A especificação pode ser um documento escrito, um conjunto de modelos gráficos, um modelo matemático formal, um conjunto de cenários de modelos de caso casos de uso, um protótipo ou qualquer combinação desses artefatos. A especificação é também a forma de comunicação sistemática entre analistas e projetistas do software. [4]

Validação: após a criação e evolução dos artefatos de requisitos, estes devem ser validados pelo grupo de usuários/clientes, assegurando que a especificação construída atende às necessidades e objetivos que motivaram 0 processo de desenvolvimento. Esta etapa tem o objetivo de assegurar que o software especificado atende às necessidades, e que as especificações podem ser cumpridas. Na validação, é examinada a qualidade das especificações; deve-se garantir que todos os requisitos do sistema foram estruturados de maneira não ambígua, que as inconsistências, omissões e erros foram apagados e corrigidos, e que os produtos de trabalho estão em conformidade com os padrões estabelecidos para o processo de desenvolvimento, para o projeto e para o produto. $[4,6]$

Gerenciamento de Requisitos: os requisitos de um software podem mudar ao longo da vida de um sistema. Novos requisitos surgem e há alterações nos requisitos em todos os estágios do processo de desenvolvimento do sistema. São comuns os casos em que mais da metade dos requisitos são alterados antes que o sistema seja posto em operação; isto causa sérios problemas para os desenvolvedores. 0 gerenciamento de requisitos corresponde ao conjunto de atividades que auxilia a equipe do projeto a identificar, controlar e rastrear os requisitos, bem como as alterações nos requisitos nos diferentes momentos do projeto. A principal função da gestão de requisitos é gerenciar as mudanças nos requisitos acordados, os relacionamentos entre os requisitos, e as dependências em ter os documentos de requisitos e outros documentos produzidos ao longo do sistema e do processo de engenharia de software. [4]

\subsection{O Ensino de Engenharia de Requisitos}

A Engenharia de Requisitos é um campo relativamente novo Engenharia de Software. Porém, tanto acadêmicos quanto profissionais vêm demonstrando um interesse particular na ER, porque é uma das subáreas mais importantes, desafiadoras e problemáticas do desenvolvimento de software. Nos últimos anos, tem havido uma ênfase na incorporação de ER aos currículos universitários da graduação. [19]

O ensino de ER deve partir do princípio que o aluno tem que se familiarizar com a resolução de problemas, metodologias e ferramentas. Isto permite ampliar a visão do aluno sobre o desenvolvimento de software durante o processo educacional de engenharia de requisitos; essas características são semelhantes ao mercado de trabalho; assim, em um projeto de negócio o aluno poderá estar mais bem preparado. [20]

Um dos desafios relevantes do ensino de ER é preparar os alunos para a elicitação de requisitos e atividades no curto tempo disponibilizado pelos cursos de graduação. A disciplina requer um aprofundamento das técnicas que permitirão futuramente que o aluno solucione problemas em diferentes cenários. [19]

O ensino da disciplina de Engenharia de Requisitos se potencializa quando, durante 0 seu ensino/aprendizagem, ocorre transferência de informações entre alunos e professor com relação às 
experiências vividas em problemas e soluções reais no mercado de trabalho. [21]

\section{Procedimentos de Pesquisa}

A pesquisa deste trabalho foi realizada através de 4 passos: Levantamento de Universidades, Investigação de sites, Análise de grade curricular e Análise dos resultados. Cada um deles é detalhado a seguir.

\subsection{Levantamento de Universidades}

Para iniciar a pesquisa desse trabalho foi preciso listar o nome de todas as universidades federais, estaduais, e institutos federais de ensino do Brasil de ensino superior. Essa etapa de construção da lista foi a base da pesquisa, que delimitou o seu tamanho e direcionou a coleta de dados.

Após montar a lista, mediante o primeiro levantamento, foi criada uma tabela, organizada e estruturada para a iniciação do processo de inserção dos resultados. Isto permitiu e facilitou a análise estatística, com o preenchimento das informações coletadas referentes às instituições de ensino a serem investigadas.

\subsection{Investigação dos Sites}

Com a influência da tecnologia, a forma de pesquisa mais utilizada é a Internet. As instituições de ensino, têm em seus sites hoje as informações que representam o seu cartão de visita. Por isso, elas buscam ter um site disponível com informações e dados precisos e funcionais. Para as instituições de ensino que têm como objetivo oferecer um processo de aprendizagem completo, dinâmico e eficiente, por intermédio de recursos tecnológicos, é essencial dispor de um sistema robusto online que atenda às necessidades para se ter cursos online de qualidade.

Com base nessas considerações, na pesquisa realizada neste artigo, todas as instituições de ensino consideradas dispõem de um site/sistema online, embora nem todas ofereçam o método de ensino EAD.

Foi realizado o processo de investigação nos sites de cada instituição de ensino para saber e ter um quantitativo de quais oferecem curso superior na modalidade de EAD. Como resultado, observou-se que mais da metade das instituições tem a modalidade EAD como forma de ensino de curso superior. Em cima dessas instituições, através de cada site, foi examinada a grade curricular dos cursos oferecidos na modalidade EAD, observando-se se dentre os disponíveis existia algum na área de TI, cursos onde geralmente é abordado o processo de desenvolvimento de software.

\subsection{Análise da Matriz Curricular}

De acordo com Rasco (1996), o currículo foi concebido como uma forma de organização e um instrumento de eficiência social, isto é, uma estrutura organizativa imposta por autoridades educativas para 'ordenar' a conduta da escolaridade. [22]

Essa etapa foi executada durante o levantamento, explorando o site de cada instituição de ensino da lista. Ainda no site, na seleção de cada curso de TI à distância, foi analisado se a grade curricular do curso estava disponível; caso afirmativo, foi verificado a existência da disciplina de Engenharia de Requisitos nessa grade curricular ou se alguma outra disciplina (semelhante) abordava os assuntos da ER. Com as informações coletadas foi alimentada a tabela relacionada com os dados da pesquisa.

\subsection{Análise dos Resultados}

Após a coleta de dados, a fase seguinte foi a de análise que teve como objetivo organizar e considerar os dados de tal forma que possibilitasse 0 fornecimento de respostas aos questionamentos da investigação. O acompanhamento dos dados incluiu uma atividade reflexiva que resultou num conjunto de informações para análise que guiaram o processo. Esse acompanhamento possibilitou registrar 0 processo e constituem importante ajuda para o desenvolvimento conceitual. [23]

A análise foi feita considerando os resultados quantitativos dos colhidos alimentados na tabela.

\section{Resultados Encontrados}

O aumento da oferta e procura de vagas, nas universidades públicas federais e estaduais no ensino superior do Brasil, tem vários motivos, entre eles temse: custos reduzidos, a excelente avaliação dos cursos destas instituições, e a exigência dos professores concursados, a valorização do mercado de trabalho, para quem tem formação acadêmica nessas instituições. Todos esses fatores ajudam a conseguir melhores propostas de trabalho. Esses são uns dos motivos pela qual são as faculdades mais bem vistas e que são referência de qualidade no Brasil. 
Pelos motivos expostos, esta pesquisa fez o recorte considerando apenas as instituições de ensino públicas descritas, delimitando assim os objetos de pesquisa. Dessa forma, pode-se verificar quantas dessas instituições oferecem ensino superior à distância, os resultados podem ser vistos em o anexo, e são resumidos a seguir.

\section{Quadro 1:}

Objeto de pesquisa: 143 instituições públicas de ensino superior (federais e estaduais) e institutos federais de ensino do Brasil.

Objetivo: levantamento do ensino na modalidade à distância na disciplina de Engenharia de Requisitos.

De acordo com o levantamento realizado, conforme gráfico apresentado na Figura 1, das 143 instituições de ensino investigadas a maioria já aderiu à modalidade EAD. Os motivos para a expansão do EAD nas instituições federais e estaduais, além da facilidade da conexão banda larga mais acessível a todos, são as vantagens que o aluno tem no EAD de: poder estudar onde e quando quiser, sem ter que estar em uma sala de aula num determinado horário, podendo estudar num horário que for mais conveniente, podendo assim organizar seu tempo e conciliar o curso com outras atividades. Além das mensalidades dos cursos à distância serem mais acessíveis ou até serem isentas, a depender da instituição de ensino, tem a economia com deslocamentos diários (transporte público, gasolina, estacionamento) e alimentação. [24]

\section{Instiuições com EAD}

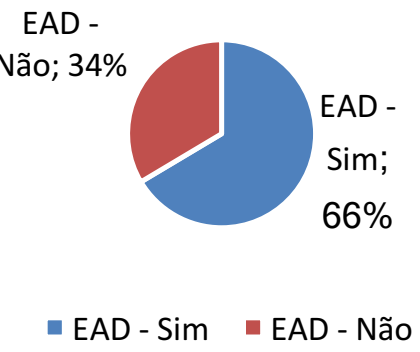

Figura 1: Instittuições de ensino com modalidades EAD (universidades federais, estaduais e institutos fedarerais). Fonte: O autor.

\subsection{Ensino EAD na Disciplina de ER}

A Figura 2 mostra um levantamento do cenário geral das universidades federais e estaduais, e institutos federais de ensino do Brasil que têm a modalidade de ensino à distância e oferecem cursos de TI e que abordam o assunto de ER em sua grade curricular de ensino.

Das instituições que oferecem cursos EAD, apenas 15 instituições (porcentagem de 17\%) têm algum curso superior de TI, que são cursos com ênfase na construção elaboração de sistemas, na sua grade de cursos oferecidos, totalizando 17 cursos de TI. Destes cursos de TI, não foi encontrado nenhuma disciplina com a nomenclatura "Engenharia de Requisitos", porém as disciplinas que foram encontradas na pesquisa possivelmente abordam a ER, pois são disciplinas que consideram de forma sistemática 0 desenvolvimento e manutenção no processo de software. Estas disciplinas contemplam 9 de Engenharia de Software e 4 de Fundamentos de Engenharia de Software.

Além disso, para 5 (cinco) cursos não foi encontrada disponível a grade curricular nos sites das respectivas instituições de ensino.

As habilidades e tarefas relativas à Engenharia de Software são organizadas em disciplinas ou áreas de conhecimento. Porém, não há em consenso ou um referencial único sobre quais sejam essas disciplinas. [24]

Segundo [24], a disciplina de Engenharia de Software é definida como um meio de criar categorias de atividades, dividida em grupos de disciplinas de engenharia, são elas:

$$
\begin{aligned}
& \text { - Modelagem de Negócio } \\
& \text { - Engenharia de Requisitos } \\
& \text { - Inálise de Projeto } \\
& \text { - Enplementação } \\
& \text { - Implantação de Testes }
\end{aligned}
$$

Um motivo de muitas vezes não se ter uma disciplina específica para a ER, é pelo fato de que a grade das disciplinas de um curso geralmente é mais enxuta possível, contendo o mínimo de tempo para abordar os assuntos para se ensinar um curso de TI. Porém a ER está presente na disciplina de Engenharia de Software, onde se tem o uso sistemático e repetitivo de técnicas para cobrir as atividades de obtenção, documentação e manutenção de um conjunto de requisitos para software que atendam aos objetivos de negócio e sejam de qualidade. Assim, a ER se insere no âmbito da Engenharia de Software, 
independentemente de qual referência em suas definições.

Com o intuito de obter um resultado mais refinado na pesquisa e obter mais informações, foi enviado um questionário via e-mail com cinco perguntas objetivas para os e-mails dos oito coordenadores dos cursos de TI. Os contatos dos coordenadores foram localizados através do site de cada instituição de ensino, uma vez que são eles mesmos que participam ativamente na elaboração curricular e metodologia do ensino das disciplinas a serem abordadas em um determinado curso.

As perguntas enviadas pretendem ajudar a entender melhor, na visão dos coordenadores e das instituições, como são montadas e estruturadas as ementas da disciplina ou os assuntos de ER, o formato como é lecionada, a forma de comunicação dos docentes com os discentes e o nível de satisfação de dos coordenadores com relação aos métodos de ensino da disciplina de ER. As cinco perguntas enviadas e o motivo de cada uma delas estão exibidos abaixo:

$1^{\text {a) }}$ "Vocês abordam assuntos de Engenharia de Requisitos em alguma disciplina?"

Para saber se os assuntos relacionados a disciplina de Engenharia de Requisitos são abordados em algum momento do curso

\section{2a) "Quais assuntos?"}

Um complemento da primeira pergunta, para ser respondido quais os assuntos de Engenharia de Requisitos são tratados durante o ensino do curso.

\section{$3^{\text {a }) ~ " E m ~ q u a i s ~ d i s c i p l i n a s ? " ~}$}

Para descobrir o nome das disciplinas que tratam dos assuntos referente a Engenharia de Requisitos

$4^{a}$ ) "Como esses assuntos são trabalhados com os alunos? Por exemplo, videoaula, projeto, questões de múltipla escolha, leitura de artigos, fórum de discussão, etc.?"

Para saber quais são os métodos de ensinoaprendizagem utilizados pela instituição de ensino para abordar, se comunicar, interagir, expor aos alunos os materiais relativos a ER.

$\left.5^{a}\right)$ "Quão satisfeito você está com o tratamento da engenharia de requisitos neste curso (escala de 1 a 5, sendo 1 péssimo e 5 ótimo)?"

Foi uma pergunta pessoal ao coordenador em relação ao nível de satisfação de como está sendo tratado e visto os assuntos referente a Engenharia de Requisitos, e para ajudar na resposta foi mostrado uma escala entre 1 à 5 .
Apenas duas instituições de ensino responderam ao e-mail, e a ambas informaram que abordam o assunto de ER durante o curso e na disciplina de Engenharia de Software. Com relação ao nível de satisfação, numa escala de 1 a 5 , uma das instituições avaliou o ensino de ER com nota 4, a outra instituição avaliou com nota 3,5 alegando não ter como alocar mais carga horária devido à restrição de tempo.

\section{Conclusões}

Ensino à distância é uma alternativa de ensino onde a metodologia é voltada para autoaprendizagem organizada e sistemática; nela o aluno, a partir do material de estudos disponível, é supervisionado por um professor/tutor distante geograficamente, e avaliado por um professor (ou grupo de professores) que se comunica por softwares pela internet. A EAD é uma metodologia que permite lecionar para um maior número de estudantes ao mesmo tempo, sem reduzir a qualidade de ensino, partilhando conhecimentos, habilidades e atividades através de um planejamento de conteúdo, trabalhos e exercícios gerenciado por uma plataforma online. Assim, é uma forma prática de ensinar e aprender. [13]

De acordo com a pesquisas realizadas, a maioria das instituições pesquisadas oferecem EAD, demonstrando a sua grande aceitação. O EAD atende a pessoas que se adaptam melhor a essa modalidade de ensino. Os fatores motivadores variam, incluindo aspectos como: comodidade, flexibilidade de horários, o reconhecimento do curso (tal qual um curso presencial), programas do governo já oferecem bolsa de estudos, a acessibilidade de banda larga para quem mora longe de alguma instituição de ensino.

Dessa forma é extremamente importante contemplar a ER nesse contexto e buscar melhores formas de ensiná-la. A importância é plenamente justificada pela relevância do processo da ER, a deficiência atual na especificação dos requisitos de sistemas no mercado, além da dimensão de assuntos abordados no ensino do conteúdo, e a necessidade de execução de atividades usando técnicas sistemáticas para ajudar na elaboração da documentação e manutenção em cenários diferentes, para que o aluno se familiarize com a resolução de problemas.

Assim, este trabalho foca no levantamento do ensino à distância na área da Engenharia de Requisitos em órgãos de ensino públicos federais e estaduais do Brasil na modalidade à distância, dando ênfase no conteúdo de ER. A motivação para a pesquisa foi ter o máximo de informações sobre $E A D$, no contexto desse domínio, para se ter conhecimento de onde e como é realizado o ensino do assunto de ER à distância. A ideia principal foi ter informação que 
desse subsídio para entender como a ER é ministrada (na estrutura e organização dos cursos com foco na área de desenvolvimento de sistemas nas instituições de ensino) e qual as técnicas utilizadas para abordar o assunto. Devido à falta de informação nos sites das instituições (como a matriz curricular e contato dos coordenadores dos cursos) e a falta de retorno dos coordenadores aos e-mails enviados, os resultados colhidos não foram tão expressivos e representativos como esperado.

A partir do que foi levantado, observou-se que o conteúdo de ER não é abordado em uma disciplina integral e exclusiva, e sim, é visto como um subtópico do contexto de "Engenharia de Software", que é um contexto maior que abrange o assunto de ER.

Como trabalhos futuros propõem-se: analisar como funcionam o conteúdo da ER à distância; fazer levantamento dos cursos de ER na modalidade EAD no exterior; analisar como vêm sendo ministrados esses cursos, ver suas capacidades, experiências e problemas.

\section{Referências}

[1] Nogueira, L.: Educação a distância. Comunicação \& Educação 5, pag: 3439. 1996

[2] Comissão de Especialistas de Ensino de Computação e Informática - CEEInf, Diretrizes Curriculares de Cursos da Área de Computação e Informática, SESuMEC, 1998.

[3] Carlise, E., Zanlorenci-GPS Robert: Engenharia de Requisitos: Processos e Técnicas no Contexto Organizacional, PUC-PR, 2006.

[4] Pressman, R., Maxim B.: Engenharia de Software-8a Edição. McGraw Hill Brasil, 2016.

[5] Paula Filho, Wilson. Engenharia de Software. Vol. 2. LTC, 2003.

[6] Lopes, L.: Um Modelo de Processo de Engenharia de Requisitos para Ambientes de Desenvolvimento Distribuído de Software. Dissertação de Mestrado, POntificia Universidade Católica do Rio Grande do Sul, 2005.

[7] Preti, O.: Educação à Distância: Fundamentos e políticas. Cuiabá: EdUFMT, 2009.

[8] OLIVEIRA, Daniel. Conceito de Educação. 117
2009. Disponível em: <http://www.ebah.com.br/content/ABAAAATI8AJ /conceito-educacao $>$. Acessado em: setembro de 2016.

[9] Belloni, M.: Educação à Distância. Autores Associados, 2006.

[10] Belloni, M.: Ensaio sobre a Educação à Distância no Brasil. Educação \& Sociedade 23.78, pag: 117-142, 2002.

[11] Portal EAD, A expansão do EAD (ensino a distância) no Brasil. Disponível em:

<http://www.ead.com.br/ead/expansao-eadbrasil.html>. Acessado em: setembro de 2016.

[12] Vilaça, M.: Educação a Distância e Tecnologias: conceitos, termos e um pouco de história. Revista Magistro 2.1, 2011.

[13] Alves, L.: Educação à Distância: Conceitos e História no Brasil e no Mundo. Revista Brasileira de Aprendizagem Aberta e a Distância, pag: 8392, 2011.

[14] Portal EAD, Vantagens do ensino a distância. Disponível em:

<http://www.ead.com.br/vantagens-do-ensinoa-distancia/> . Acessado em: setembro de 2016

[15] MEC, Educação Superior a Distância. Disponível em:

$<$ http://portal.mec.gov.br/instituicoescredenciadas/educacao-superior-a-distancia $>$. Acessado em: setembro de 2016.

[16] Pressman, R.: Software engineering: a practitioner's approach. Palgrave Macmillan, 2005.

[17] Thayer, R., Sidney, B., Dorfman, D.: Software Requirements Engineering. IEEE Computer Society Press, 1997.

[18] da Silva Rocha, Rafael, and Teresinha Moreira de Magalhães. "ENGENHARIA DE REQUISITOS." Fundação Educacional São José, Revista Eletrônica, 4ª Edição, 2005.

[19] Norazlin, Y., Mehboob, Z., Zowghi, D.: The Role of Conducting Stakeholder Meetings in Requirements Engineering Training." Proceedings of REET '07 pag: 48-55.

http://dx.doi.org/10.25286/repa.v2i2.561 
[20] Memon, R., Ahmad, R. and Salim, S.: Problems in requirements engineering education: a survey. Proceedings of the 8th International Conference on Frontiers of Information Technology. ACM, 2010.

[21] Regev, G., Gause, D., Wegmann, A.: Requirements Engineering Education in the $21^{\text {st }}$ century, An Experiential Learning Approach. 16th IEEE International Requirements Engineering Conference. IEEE, 2008.

[22] Rasco, A., Félix, J. e Nieves, B; (Coord.). Teoría y desarrollo Del currículo. Ediciones Aljibe, 1996. Angulo Rasco, J. F. "¿A qué llamamos curriculum?". En: Angulo Rasco, J. F. y Blanco, N. (Comp.) Teoría y Desarrollo del Curriculum, Aljibe, Málaga, 1994, (capítulo 1, pp.17-29).

[23] Gil, A.: Métodos e técnicas de pesquisa social. Métodos e técnicas de pesquisa social. Atlas, 2010.

[24] Vazquez, C., Simões, G:. Engenharia de Requisitos: Software Orientado ao Negócio. Brasport, 2016. 


\section{Apêndice I}

\begin{tabular}{|c|c|c|c|}
\hline Órgão & Instituição de Ensino & EAD? & $\begin{array}{c}\text { Curso de } \\
\text { TI? }\end{array}$ \\
\hline Federal & Universidade de Brasília & Sim & Não \\
\hline Federal & Universidade Federal da Grande Dourados & Sim & Sim \\
\hline Federal & Universidade Federal de Goiás & Não & Não \\
\hline Federal & Universidade Federal de Mato Grosso & Sim & Não \\
\hline Federal & Universidade Federal de Mato Grosso do Sul & Sim & Não \\
\hline Federal & Universidade Federal da Bahia & Não & Não \\
\hline Federal & Universidade Federal do Sul da Bahia & Não & Não \\
\hline Federal & Universidade Federal do Recôncavo da Bahia & Sim & Não \\
\hline Federal & Uni. F. da Integração Internacional da Lusofonia Afro-Brasileira & Sim & Não \\
\hline Federal & Universidade Federal da Paraíba & Sim & Não \\
\hline Federal & Universidade Federal do Cariri & Não & Não \\
\hline Federal & Universidade Federal de Alagoas & Não & Não \\
\hline Federal & Universidade Federal de Campina Grande & Sim & Não \\
\hline Federal & Universidade Federal de Pernambuco & Sim & Não \\
\hline Federal & Universidade Federal de Sergipe & Não & Não \\
\hline Federal & Universidade Federal do Ceará & Não & Não \\
\hline Federal & Universidade Federal do Maranhão & Sim & Não \\
\hline Federal & Universidade Federal do Oeste da Bahia & Sim & Não \\
\hline Federal & Universidade Federal do Piauí & Sim & Sim \\
\hline Federal & Universidade Federal do Rio Grande do Norte & Não & Não \\
\hline Federal & Universidade Federal do Vale do São Francisco & Sim & Não \\
\hline Federal & Universidade Federal Rural de Pernambuco & Sim & Sim \\
\hline Federal & Universidade Federal Rural do Semi-Árido & Sim & Não \\
\hline Federal & Universidade Federal de Rondônia & Sim & Não \\
\hline Federal & Universidade Federal de Roraima & Sim & Sim \\
\hline Federal & Universidade Federal do Acre & Sim & Sim \\
\hline Federal & Universidade Federal do Amapá & Sim & Não \\
\hline Federal & Universidade Federal do Amazonas & Sim & Não \\
\hline Federal & Universidade Federal do Oeste do Pará & Não & Não \\
\hline Federal & Universidade Federal do Pará & Sim & Não \\
\hline Federal & Universidade Federal do Tocantins & Sim & Não \\
\hline Federal & Universidade Federal Rural da Amazônia & Não & Não \\
\hline Federal & Universidade Federal do Sul e Sudeste do Pará & Não & Não \\
\hline Federal & Universidade Federal de Alfenas & Sim & Não \\
\hline Federal & Universidade Federal de Itajubá & Sim & Não \\
\hline Federal & Universidade Federal de Juiz de Fora & Sim & Sim \\
\hline Federal & Universidade Federal de Lavras & Sim & Não \\
\hline Federal & Universidade Federal de Minas Gerais & Sim & Não \\
\hline Federal & Universidade Federal de Ouro Preto & Sim & Não \\
\hline Federal & Universidade Federal de São Carlos & Sim & Sim \\
\hline Federal & Universidade Federal de São João del-Rei & Não & Não \\
\hline Federal & Universidade Federal de São Paulo & Sim & Não \\
\hline Federal & Universidade Federal de Uberlândia & Sim & Não \\
\hline Federal & Universidade Federal de Viçosa & Sim & Não \\
\hline Federal & Universidade Federal do $A B C$ & Não & Não \\
\hline Federal & Universidade Federal do Espírito Santo & Sim & Não \\
\hline Federal & Universidade Federal do Estado do Rio de Janeiro & Sim & Não \\
\hline Federal & Universidade Federal do Rio de Janeiro & Não & Não \\
\hline Federal & Universidade Federal do Triângulo Mineiro & Não & Não \\
\hline Federal & Universidade Federal dos Vales do Jequitinhonha e Mucuri & Sim & Não \\
\hline Federal & Universidade Federal Fluminense & Não & Não \\
\hline Federal & Universidade Federal Rural do Rio de Janeiro & Não & Não \\
\hline Federal & Universidade Federal da Fronteira Sul & Não & Não \\
\hline Federal & Universidade Federal da Integração Latino-Americana & Não & Não \\
\hline Federal & Universidade Federal de Ciências da Saúde de Porto Alegre & Não & Não \\
\hline Federal & Universidade Federal de Pelotas & Sim & Não \\
\hline Federal & Universidade Federal de Santa Catarina & Sim & Não \\
\hline Federal & Universidade Federal de Santa Maria & Sim & Não \\
\hline Federal & Universidade Federal do Pampa & Não & Não \\
\hline Federal & Universidade Federal do Paraná & Sim & Não \\
\hline Federal & Universidade Federal do Rio Grande & Sim & Não \\
\hline Federal & Universidade Federal do Rio Grande do Sul & Sim & Sim \\
\hline Federal & Universidade Tecnológica Federal do Paraná & Sim & Não \\
\hline
\end{tabular}




\begin{tabular}{|l|}
\hline Estadual \\
\hline Estadual \\
\hline Estadual \\
\hline Estadual \\
\hline Estadual \\
\hline Estadual \\
\hline Estadual \\
\hline Estadual \\
\hline Estadual \\
\hline Estadual \\
\hline Estadual \\
\hline Estadual \\
\hline Estadual \\
\hline Estadual \\
\hline Estadual \\
\hline Estadual \\
\hline Estadual \\
\hline Estadual \\
\hline Estadual \\
\hline Estadual \\
\hline Estadual \\
\hline Estadual \\
\hline Estadual \\
\hline Estadual \\
\hline Estadual \\
\hline Estadual \\
\hline Estadual \\
\hline Estadual \\
\hline Estadual \\
\hline Estadual \\
\hline Estadual \\
\hline Estadual \\
\hline Estadual \\
\hline Estadual \\
\hline Estadual \\
\hline Estadual \\
\hline Estadual \\
\hline Estadual \\
\hline Estadual \\
\hline Estadual \\
\hline Estadual \\
\hline Instituto Fed \\
\hline Instituto \\
\hline
\end{tabular}

Instituto Federa

Instituto Federal

Instituto Federal

Instituto Federal

Instituto Federal

Instituto Federal

Instituto Federal

Instituto Federal

Instituto Federal

Instituto Federal

Instituto Federal

Instituto Federal

Instituto Federal

Instituto Federal

Instituto Federal

Instituto Federal

Instituto Federal

Instituto Federal

Instituto Federal

Instituto Federal

Instituto Federal

Instituto Federal

Instituto Federal

Instituto Federal

Instituto Federal

Instituto Federal

Instituto Federal

Instituto Federal

Instituto Federal

Instituto Federal

Instituto Federal

Instituto Federal
Universidade Estadual de Goiás

Universidade do Estado de Mato Grosso

Universidade Estadual de Mato Grosso do Sul

Universidade Estadual de Alagoas

Universidade Estadual de Ciências da Saúde de Alagoas

Universidade do Estado da Bahia

Universidade Estadual de Feira de Santana

Universidade Estadual de Santa Cruz

Universidade Estadual do Sudoeste da Bahia

Universidade Estadual do Ceará

Universidade Estadual Vale do Acaraú

Universidade Regional do Cariri

Universidade Estadual do Maranhão

Universidade Estadual da Paraíba

Universidade de Pernambuco

Universidade Estadual do Piauí

Universidade do Estado do Rio Grande do Norte

Universidade do Estado do Amapá

Universidade do Estado do Amazonas

Universidade Estadual do Saber Tradicional da Amazônia

Universidade do Estado do Pará

Universidade Estadual de Roraima

Universidade do Tocantins

Universidade do Estado de Minas Gerais

Universidade Estadual de Montes Claros

Universidade do Estado do Rio de Janeiro

Universidade Estadual da Zona Oeste

Universidade Estadual do Norte Fluminense Darcy Ribeiro

Universidade de São Paulo

Universidade Estadual de Campinas

Universidade Estadual Paulista "Júlio de Mesquita Filho"

Universidade Virtual do Estado de São Paulo

Universidade Estadual de Londrina

Universidade Estadual de Maringá

Universidade Estadual do Paraná

Universidade Estadual de Ponta Grossa

Universidade Estadual do Centro-Oeste

Universidade Estadual do Norte do Paraná

Universidade Estadual do Oeste do Paraná

Universidade Estadual do Rio Grande do Sul

Universidade do Estado de Santa Catarina

Instituto Federal de Brasília

Instituto Federal de Goiás

Instituto Federal Goiano

Instituto Federal de Mato Grosso

Instituto Federal de Mato Grosso do Sul

Instituto Federal de Alagoas

Instituto Federal da Bahia

Instituto Federal Baiano

Instituto Federal do Ceará

Instituto Federal do Maranhão

Instituto Federal da Paraíba

Instituto Federal de Pernambuco

Instituto Federal do Sertão Pernambucano

Instituto Federal do Piauí

Instituto Federal do Rio Grande do Norte

Instituto Federal de Sergipe

Instituto Federal do Acre

Instituto Federal do Amapá

Instituto Federal do Amazonas

Instituto Federal do Pará

Instituto Federal de Rondônia

Instituto Federal de Roraima

Instituto Federal do Tocantins

Instituto Federal do Espírito Santo

Instituto Federal de Minas Gerais

Instituto Federal do Norte de Minas Gerais

Instituto Federal do Sudeste de Minas

Instituto Federal do Sul de Minas

Instituto Federal do Triângulo Mineiro

Colégio Pedro II

Instituto Federal do Rio de Janeiro

Instituto Federal Fluminense

\begin{tabular}{|c|c|}
\hline Sim & Não \\
\hline Sim & Não \\
\hline Sim & Não \\
\hline Não & Não \\
\hline Não & Não \\
\hline Sim & Sim \\
\hline Não & Não \\
\hline Sim & Não \\
\hline Não & Não \\
\hline Sim & Sim \\
\hline Não & Não \\
\hline Não & Não \\
\hline Sim & Não \\
\hline Sim & Não \\
\hline Sim & Não \\
\hline Sim & Não \\
\hline Sim & Não \\
\hline Não & Não \\
\hline Não & Não \\
\hline Não & Não \\
\hline Sim & Não \\
\hline Não & Não \\
\hline Sim & Não \\
\hline Sim & Não \\
\hline Sim & Não \\
\hline Sim & Sim \\
\hline Não & Não \\
\hline Sim & Sim \\
\hline Sim & Não \\
\hline Sim & Não \\
\hline Sim & Não \\
\hline Não & Não \\
\hline Sim & Não \\
\hline Sim & Não \\
\hline Não & Não \\
\hline Não & Não \\
\hline Sim & Não \\
\hline Sim & Não \\
\hline Não & Não \\
\hline Não & Não \\
\hline Sim & Não \\
\hline Sim & Não \\
\hline Sim & Não \\
\hline Sim & Não \\
\hline Não & Não \\
\hline Sim & Não \\
\hline Sim & Não \\
\hline Não & Não \\
\hline Sim & Sim \\
\hline Sim & Não \\
\hline Sim & Sim \\
\hline Sim & Sim \\
\hline Sim & Não \\
\hline Não & Não \\
\hline Não & Não \\
\hline Sim & Não \\
\hline Sim & Não \\
\hline Sim & Não \\
\hline Sim & Não \\
\hline Sim & Não \\
\hline Sim & Sim \\
\hline Sim & Não \\
\hline Sim & Não \\
\hline Sim & Não \\
\hline Não & Não \\
\hline Sim & Não \\
\hline Sim & Não \\
\hline Sim & Não \\
\hline Sim & Não \\
\hline Sim & Sim \\
\hline Não & Não \\
\hline Não & Não \\
\hline Sim & Não \\
\hline
\end{tabular}


Revista de Engenharia e Pesquisa Aplicada (2017) Vol.2 No.2.

\begin{tabular}{|l|l|c|c|}
\hline Instituto Federal & Instituto Federal de São Paulo & Sim & Não \\
\hline Instituto Federal & Instituto Federal do Paraná & Sim & Não \\
\hline Instituto Federal & Instituto Federal do Rio Grande do Sul & Sim & Não \\
\hline Instituto Federal & Instituto Federal Farroupilha & Sim & Não \\
\hline Instituto Federal & Instituto Federal Sul-rio-grandense & Não & Não \\
\hline Instituto Federal & Instituto Federal de Santa Catarina & Não & Não \\
\hline Instituto Federal & Instituto Federal Catarinense & Não & Não \\
\hline
\end{tabular}

